\title{
La educación continua en radiología durante la COVID-19: más allá del aprendizaje a distancia
}

\section{Continuing education in radiology during COVID-19: beyond distance}

\section{learning}

Georgina Cornelio-Rodríguez ${ }^{1 *}$, Paloma Pérez-Ladrón de Guevara ${ }^{1}$, Juan P. Reyes-González ${ }^{1}$, José L. Ramírez-Arias ${ }^{1}$ y Roberto J. Mercado-Elizondo ${ }^{2}$

${ }^{1}$ Servicio de Radiología e Imagen, Hospital Ángeles Pedregal, Ciudad de México; ${ }^{2}$ Servicio de Radiología, Unidad Médica de Alta Especialidad de Pediatría, Centro Médico Nacional de Occidente, Guadalajara, Jalisco. México

\section{RESUMEN}

Introducción: La pandemia por COVID-19 obligó a la educación en la radiología, así como a otras especialidades, a dar un cambio súbito y adoptar nuevos recursos para el apoyo de la educación, en particular el uso de las plataformas digitales. Objetivo: Conocer los cambios implementados en el aprendizaje de los radiólogos y los médicos en formación a través de las soluciones tecnológicas. Material y método: Se utilizaron medidas de estadística descriptiva como promedio, moda y mediana para el análisis de la muestra. Resultados: Se obtuvieron respuestas de 182 personas y se analizaron las características de las clases virtuales, como el tipo de plataforma más utilizado, representado por Zoom con una preferencia del 70\% de los encuestados; se identificó que la falta de retroalimentación y la interacción fueron las características negativas referidas con más frecuencia. Conclusiones: Este trabajo demuestra el carácter apreciativo del aprendizaje en línea, que ha sido instituido sin trabajos previos que demuestren su utilidad, y los aspectos negativos debido a la falta de análisis y su aplicación repentina. Se ha demostrado en este trabajo una apreciación notablemente menor del aprendizaje, afectado por la falta de interacción, retroalimentación y disminución de la calidad global cuando se compara con las clases presenciales.

Palabras clave: Educación. Radiología. Aprendizaje. COVID-19. 


\section{ABSTRACT}

Introduction: The COVID-19 pandemic conditioned that education in radiology, as well as with other specialties, gave a sudden change where to implement new resources to support education, mainly the use of digital platforms. Objective: To know the changes implemented in the learning of radiologists and doctors in training through technological solutions. Material and method: Descriptive statistics measures such as mean, mode and median were used for the analysis of the sample. Results: Answers were obtained from 182 people, analyzing the characteristics of the virtual classes, such as the type of platform most used, represented by zoom with a preference of $70 \%$ of the respondents, finding the lack of feedback and the lack of interaction as the negative characteristics mostly referred to. Conclusions: This work demonstrates the appreciative nature of online learning, which, due to the lack of analysis and its sudden application, has been implemented without previous work that demonstrates its usefulness and negative aspects, demonstrated in this work as a remarkably appreciation less learning, affected by the lack of interaction, feedback and decrease in global quality when compared to face-to-face classes.

Key words: Education. Radiology. Learning. COVID-19.

\section{INTRODUCCIÓN}

La Organización Mundial de la Salud declaró la infección por COVID-19 como una emergencia sanitaria pública de ámbito mundial el día 30 de enero de 2020 y el 11 de marzo como una pandemia.

Ya ha pasado un año desde el inicio de esta infección de carácter mundial y no desaparecerá en los siguientes meses e incluso quizá años. El aislamiento social y las medidas de confinamiento han permitido atenuar nuevas infecciones, las cuales se han desarrollado en forma escalonada y lenta en esta nación. Esto obligó a implementar también en los servicios de radiología e imagen nuevas medidas de distanciamiento que afectaron el programa de enseñanza o educación continua necesario para los médicos radiólogos y los futuros radiólogos en formación ${ }^{1}$.

Esta crisis mundial tiene todavía un efecto notable en la enseñanza médica, dado que las actividades internas y externas se afectaron en cuanto a la organización de las sesiones ya programadas, la cancelación de cursos o congresos y la modificación de los planes de estudio previamente planeados o las simples actividades diarias del servicio de imagen.

\section{EDUCACIÓN CONTINUA EN RADIOLOGÍA}

En todas las instituciones, los departamentos de radiología se vieron en la necesidad de instituir rápidamente soluciones tecnológicas que permitieran a los radiólogos contribuir de forma 
remota en lugares de trabajo distantes, o en algunos casos desde los hogares, incluidas las modificaciones en los protocolos de exámenes, interpretación, discusiones de casos multidisciplinarios, evaluaciones y educación continua.

La solución tecnológica más rápida fue la implementación de educación en línea, pero ésta requiere motivación y perseverancia reales para aprender de forma individual; esto, más que representar un obstáculo, es una característica. Debido a las condiciones en las que se han desarrollado las elevaciones de los contagios del COVID-19 en este país, la educación en línea podría durar muchos meses más como la principal solución para impedir la exposición al contagio, más aún como radiólogos, lo que representa una buena prueba de algunas de las cualidades que son deseables en los médicos: persistencia, determinación y compromiso con el aprendizaje permanente ${ }^{2}$.

\section{FORMACIÓN DE EQUIPOS}

La emergencia sanitaria dio lugar a que los departamentos de control de infecciones en los diferentes hospitales adoptaran el aislamiento del personal sintomáticos y el distanciamiento social para reducir la transmisión de los portadores asintomáticos; dentro de estas medidas de mitigación de riesgos se incluyó separar las estaciones de trabajo por al menos $1.5 \mathrm{~m}$, reducir el número de citas, consultas y conferencias, así como rotar al personal con la formación de equipos dentro del servicio donde los integrantes no debían interactuar entre sí, de tal modo que si alguno de sus miembros se convertía en un caso confirmado o probable de COVID-19, el resto del equipo podía seguir su trabajo, es decir, el objeto era contener el contagio ${ }^{3,4}$.
Sin embargo, para los residentes de radiología estas medidas significaron una menor concentración del trabajo habitual, lo que representó no permanecer en el servicio en todos los casos, debido a la mayor necesidad de flexibilidad por estas medidas de prevención ${ }^{5}$.

Es realista afirmar que las instituciones de enseñanza no estaban preparadas para un problema de estas dimensiones, por lo que se llevó a cabo un despliegue acelerado de soluciones de educación a distancia para asegurar la continuidad pedagógica; tal y como Giannini (2020) refiere: "Los obstáculos son múltiples, desde la baja conectividad y la falta de contenido en línea alineado con los planes de estudio hasta un profesorado no preparado para esta nueva normalidad."6

\section{REDISTRIBUCIÓN}

En algunos centros hospitalarios, los médicos radiólogos y los residentes en formación se redistribuyeron dentro de las áreas COVID (unidades de cuidados intensivos) o de triage, todos entornos de atención directa al paciente. Esta redistribución fue quizá un área de oportunidad para unirse a los clínicos de otras especialidades. Sin embargo, esta redistribución a entornos no radiológicos debió realizarse de forma voluntaria, en la medida de lo posible ${ }^{4}$.

\section{INTERACCIÓN CON PACIENTES}

La interacción con los pacientes dentro de la radiología tiende a ser limitada; en consecuencia, para protección del personal médico y de los pacientes, muchos servicios de imagen tuvieron que posponer sus estudios e 
incluso diferir algunos procedimientos intervencionistas electivos; la prioridad fue la continuidad de los servicios de urgencia, ya que el acceso al equipo de protección personal (EPP) y la capacitación sobre el uso adecuado del EPP fueron cruciales, en virtud de la escasez y para la propia seguridad de los médicos radiólogos; estos últimos han sido blanco fácil durante esta pandemia debido a que son uno de los principales filtros de diagnóstico del COVID-194.

La interacción atenuada con los pacientes dio lugar a que esa parte importante de la clínica incidiera en los diagnósticos o las posibilidades diagnósticas posibles.

\section{EVALUACIÓN}

Otro punto importante es la evaluación, es decir, el proceso administrado por el profesorado adjunto a la institución y acorde a los criterios y niveles dispuestos; en este punto se requieren las actividades en las que el residente demuestre sus logros de aprendizaje y son los aspectos o cualidades los que determinan el nivel de calidad que debe exigirse. El clínico es orientado a recoger información y evidencias para emitir un juicio de valor sobre la calidad de un determinado logro. En el caso de la radiología, se basa en la interpretación de imágenes radiológicas, la anatomía, la semiología radiológica y la patorradiología, esto es, los criterios semiológicos que se definen en una imagen radiológica. La valoración del aprendizaje también debe incluir una adecuada retroalimentación (feedback), ya que es importante reflexionar sobre los resultados?.

\section{COMPETENCIAS EN RADIOLOGÍA}

El término "competencia" procede del latín y se refiere a la aptitud o idoneidad para hacer algo ${ }^{8}$ El médico radiólogo Ros Mendoza (2017) lo define como el conjunto de habilidades cognitivas, destrezas motoras, actitudes y valores, conductas o comportamientos que hacen posible llevar a cabo, de manera eficaz, cualquier actividad. El resultado del aprendizaje es una consecuencia medible y manifiesta el grado de desarrollo en la competencia. El temario no puede ser el centro de la planificación porque éste se determina con los resultados del proceso de aprendizaje, es decir, la actividad que realiza el estudiante. Los mejores programas de actividades son aquellos en los que el estudiante tiene mayor participación, en los que se encuentra más activo.

La evaluación de las competencias que debe tener un médico residente en radiología podría resumirse con las siguientes preguntas:

- ¿Cuál es el estudio mostrado?

- ¿Cuáles son las estructuras anatómicas observadas?

- Definición de las características del estudio: técnica, contraste, reconstrucciones, etc.

- Signos radiológicos presentes.

- ¿Con este estudio se puede establecer un diagnóstico?

- ¿Cuál es la mportancia clínica de los hallazgos?

- Consideración de los diagnósticos diferenciales: ise requieren otros estudios de extensión para establecer el diagnóstico definitivo? ${ }^{7}$ 


\section{REORGANIZACIÓN DEL SERVICIO}

Como ya se mencionó, con la formación de equipos y la redistribución de los integrantes del servicio de imagen se introdujo un cambio importante en la oferta formativa externa de los residentes y los radiólogos; esto supuso cancelaciones de cursos y congresos y ello produjo efectos negativos en la formación continua y los programas de residencia médica. Lo anterior es un golpe directo a la formación práctica, ya que también condujo a la anulación de las prácticas internas y la cancelación de las rotaciones en los campos clínicos externos. En este sentido, debe considerarse la utilización de los simuladores.

En lo que respecta a la parte teórica, se compensa con el uso de sistemas de gestión del aprendizaje en plataformas (learning management system), por lo que el efecto tiende a ser menor. En la parte práctica, el tema se complica desde el comienzo de la organización, ya que la principal dificultad en situación de pandemia se limita por las medidas de seguridad $^{1}$.

Esto obliga a adecuar y rediseñar las rotaciones de muchos médicos residentes, sobre todo en los espacios más restringidos, como el de la resonancia mágnetica, el procesamiento de imágenes en las estaciones de trabajo de tomografía y los procedimientos en las salas de intervención, sobre todo en aquellos residentes que se encuentran en rotación en esos servicios por primera vez. Todo ello se explica por la importancia de mantener un espacio seguro, dado que la probabilidad de contagio aún es elevada.

\section{INVESTIGACIÓN}

Muchos protocolos de investigación se han suspendido o pausado debido a la restricción del acceso de los pacientes participantes a las instituciones con el fin de no propiciar la cadena de contagio; en consecuencia, han disminuido las oportunidades de colaboración y trabajo en red ${ }^{4}$.

Sin embargo, realizar investigaciones descriptivas o retrospectivas puede ser una adecuada metodología para revisar otros proyectos $y$ trabajar en ellos durante la pandemia, para no perder el valor del trabajo. Muchos profesores investigadores de la radiología disponen ahora de tiempo para concluir los proyectos en curso, por lo que también la crisis económica tiene un efecto considerable en el desarrollo de los programas de investigación. Por consiguiente, a pesar de las dificultades para la investigación, es necesario tratar de obtener becas para proyectos específicos ${ }^{1}$.

\section{CERTIFICACIÓN POR EL CONSEJO}

El Consejo Mexicano de Radiología e Imagen es el organismo encargado de certificar a los médicos radiólogos al término de su especialidad y renovar su certificación cada cinco años sobre la base de actualizaciones de su actividad académica y la verificación de la actualización continua de los radiólogos. La pandemia también modificó las fechas de aplicación de los exámenes de certificación y tuvo la necesidad de cerrar sus oficinas en forma temporal con motivo de la aplicación del semáforo rojo en dos ocasiones como medida preventiva, indicada por las autoridades gubernamentales. Esta institución se vio obligada 
a ofrecer atención por vía telefónica o por correo electrónico y todos los trámites de registro debieron realizarse en su plataforma en línea. Cuando reanudó sus actividades, tuvo que acoplarse en su totalidad a las medidas sanitarias establecidas. En cuanto a las certificaciones, sólo se prolongaron en relación con las fechas previstas; sin embargo, proporcionó tutoriales en cuanto a la aplicación de los exámenes, además de especificar las características que el médico debía incluir en sus equipos y el sistema de vigilancia para realizar sus certificaciones en tiempo y forma; el propio consejo mencionó que a partir de esta pandemia la forma de examinar ha sido ya parte de la historia porque los exámenes se efectuaron desde los hogares del aplicante, lo cual representó un gran trabajo para el comité de evaluación ${ }^{9}$.

\section{CURSOS DE RADIOLOGÍA Y ALTA ESPECIALIDAD}

Todos los cursos de radiología general se han afectado de cierta forma por la pandemia, toda vez que sus programas sufrieron modificaciones de manera emergente y se ajustaron a su vez a las posibles bajas temporales de sus integrantes en caso de contagio por COVID-19. Si se considera que la práctica y supervisión dirigida son parte fundamental en la especialidad, el decremento del número de pacientes del que dispone con regularidad un servicio de radiología fue también un determinante para el aprendizaje, pese a lo cual debe reconocerse que uno de los elementos más notables era tratar de atenuarlo con el proceso de retroalimentación.

Por lo tanto, había que mantener y desarrollar sesiones clínicas, además de hacer una adaptación de acuerdo a las condiciones para que en las sesiones pudieran participar todos los miembros del servicio. De igual modo, fue necesario favorecer el uso de las herramientas virtuales para los nuevos modelos de actividades formativas, y con horarios más flexibles, para que los cursos impartidos por ciertas instituciones o empresas aportaran puntos curriculares y todos los integrantes pudieran integrarse y participar. Todas las instituciones deben resaltar la importancia de mantener programas de formación, continuada en el ámbito de la infección por COVID-19, en particular en relación con los hallazgos radiológicos, pero también con otros temas propios de la radiología general, ya que el resto de las enfermedades sigue presente al margen de la pandemia ${ }^{1,5}$.

El uso de los simuladores multifuncionales es una de las alternativas para la radiología simple y para la exploración por tomografía computarizada, muchos de ellos diseñados para la evaluación de las imágenes de estudios angiográficos por tomografía y que pueden emplearse como herramienta docente para la interpretación de imágenes médicas. Asimismo, existen otros simuladores en el área del ultrasonido y el intervencionismo percutáneo que hacen posible identificar y localizar nódulos y quistes, así como realizar biopsias guiadas; esto permite desarrollar habilidades al simular procedimientos de principio a final, aunque también están disponibles entrenadores para la colocación de accesos venosos periféricos ${ }^{10}$. Existe un simulador importante en el área del intervencionismo, el CathLabVR, que es un sistema modular portátil que permite a los residentes y los médicos llevar a cabo procedimientos de cateterismo en cualquier lugar; con este instrumento es posible navegar por diversas anatomías virtuales en 3D, incluidas anomalías vasculares 
periféricas y cardíacas, a medida que los clínicos desarrollan competencia dentro de un entorno de aprendizaje seguro ${ }^{11}$. Además de estos ejemplos que son los más frecuentes, existen otros simuladores que pueden ayudar en la práctica de la formación médica.

El objetivo primordial es continuar la formación de estudiantes y médicos residentes con la misma calidad que hasta ahora o superior mediante las herramientas digitales en este nuevo entorno.

\section{CURSOS Y CONGRESOS}

Esta pandemia afectó la organización de cursos y congresos. De manera inicial, las medidas se consideraron provisionales, pero luego se cambiaron o aplazaron las fechas de las actividades e incluso de cancelaron. Sin embargo, también los problemas económicos, la carga de trabajo mayor para los próximos meses y las incertidumbres relacionadas con la evolución de esta infección hacen que la forma de organizar las actividades formativas y las reuniones científicas experimenten cambios en verdad drásticos. El uso de las plataformas en línea ha tratado de compensar de cierta forma estas deficiencias. Cabe señalar que muchos de estos cursos y congresos en línea han apoyado los créditos curriculares para la certificación y la recertificación ${ }^{1}$.

\section{USO DE LAS PLATAFORMAS LMS ILEARNING MANAGEMENT SYSTEM) Y OTRAS HERRAMIENTAS ELECTRÓNICAS}

La integración digital que se expande de modo global también se observa en el sector salud, particularmente en el área de la enseñanza, un fenómeno que se aceleró con esta pandemia. El uso de plataformas digitales es un claro ejemplo de esta traslación ${ }^{12,13}$.

El LMS o sistema de gestión de aprendizaje puede definirse como una plataforma de software alojada en la red que proporciona un entorno de aprendizaje interactivo en línea ${ }^{14}$. Estos sistemas podrían clasificarse en términos generales como pertenecientes a uno de dos campos: de paga o de código abierto. Sin embargo, en uno u otro caso debe ser capaz de ejecutar reversas funciones, entre ellas la capacidad de difundir conocimientos, la evaluación de la competencia del usuario, el registro de los logros del usuario, el soporte de herramientas de comunicación (síncronas y asíncronas) y la seguridad del sistema.

Varios estudios han comparado los métodos de enseñanza electrónicos y tradicionales en estudiantes de diversas facultades de medicina y han demostrado que el aprendizaje asistido por electrónicos se relaciona con la mejoría en calificaciones, así como la capacidad de resolución de problemas ${ }^{15}$. La radiología ofrece una gran oportunidad para aprovechar los avances tecnológicos para la educación por su naturaleza inherentemente digital. En un estudio de Sparacia, et al. se observó que en el ámbito médico, de modo específico el radiológico, estas plataformas se han utilizado como fuentes de consulta para dar respuesta a preguntas clínicas, preparar ponencias y difundir sesiones de aprendizaje a distancia, entre otros ${ }^{14,15}$.

Uno de los sistemas de gestión de aprendizaje ampliamente conocido es Radiopaedia. Esta plataforma aloja una gran cantidad de 
artículos que tienen como finalidad ofrecer una breve revisión de varios temas desde un enfoque radiológico; además, incluye más de 40 mil casos en imágenes, cursos de diferentes áreas de la radiología (p. ej., radiología de urgencias, neuroimagen, etc.), así como múltiples exámenes de práctica (Fig. 1) ${ }^{16}$.

Ctisus es una plataforma dedicada a la imagen por tomografía que fundó el profesor Eliot K. Fishman en la que puede accederse a lecturas, casos y exámenes, y dispone además de múltiples aplicaciones para descargar en dispositivos móviles de diferentes temas, como atlas de tórax, patología de la columna vertebral, listas de verificación de masas suprarrenales, entre otros (Fig. 2) ${ }^{17}$.

Otra de las plataformas más consultadas es STATdx, la cual gestiona la editorial Elsevier y cuya finalidad es poner al alcance de radiólogos en formación recursos que faciliten el proceso diagnóstico. En su página web se pueden encontrar más de 4,000 revisiones de temas a manera de diagnósticos, más de 1,300 imágenes, al menos 20,000 casos y 195 procedimientos. En los temas de diagnóstico se incluye información para ayudar a tomar decisiones críticas, por ejemplo diagnósticos diferenciales, galerías de imágenes para referencia y una breve revisión de la anatomía ${ }^{18}$.

La International Society of Radiology tiene a disposición una plataforma conocida como International Commision on Radiology Education en la que se pueden encontrar cursos gratuitos, webinars, lecturas, casos, libros, entre otros ${ }^{19}$.

Por su parte, las sociedades de radiología mundiales se sumaron al esfuerzo de promover diferentes actividades académicas de

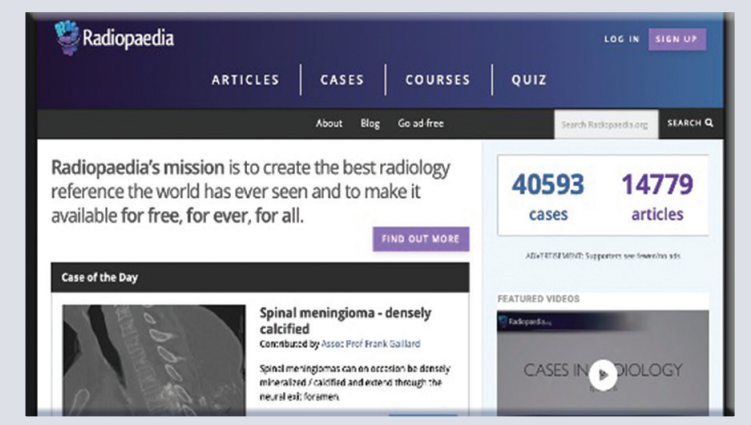

Figura 1. Página de inicio de Radiopaedia.

manera virtual, al margen de la labor académica que ya desarrollaban.

El Colegio Interamericano de Radiología, en solidaridad con la comunidad radiológica, y a través del portal de su departamento de educación, puso a disposición todos sus cursos virtuales (un total de 73) que abarcan temas de diagnóstico por imagen de áreas diversas, como sistema musculoesquelético, neurorradiología, oncología. Más información puede consultarse en http://campusradiologiavirtual.org/\#cursosgratuitos ${ }^{20}$

Durante las primeras semanas de la pandemia, la European Society of Radiology (ESR) puso a consulta libre de todos sus miembros, por primera vez de manera completamente gratuita, su contenido sobre demanda a través de ESR Connect (https://connect.myesr.org) con acceso a múltiples cursos, entre ellos los de imagen cardíaca e imagen del tórax, cabeza y cuello. Además, como muchas otras sociedades decidió celebrar su congreso anual de forma virtual, con conferencias en vivo y sobre demanda. En la actualidad apoya el crecimiento de médicos radiólogos y radiólogos en formación a través de cursos sobre demanda ${ }^{21}$. 


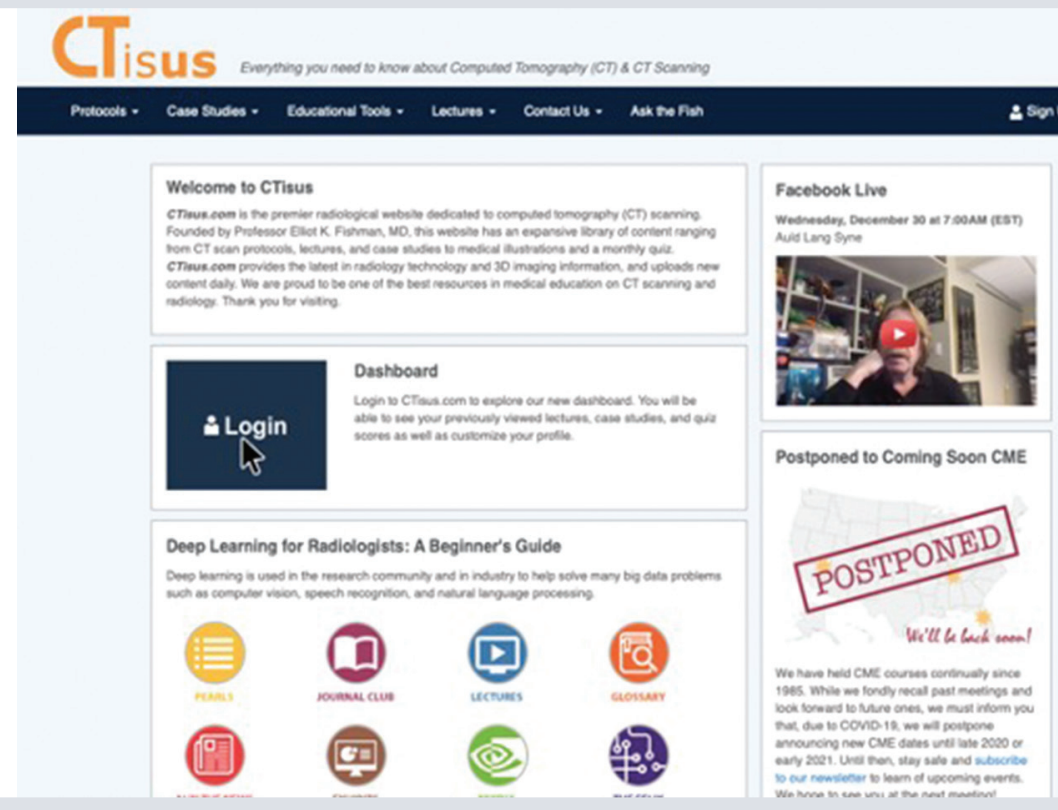

Figura 2. Página de inicio de CTisus.

Este año, la Radiology Society of North America (RSNA) transmitió su $106^{\mathrm{a}}$ Asamblea y Reunión Anual de manera absolutamente virtual. Durante siete días, médicos radiólogos y radiólogos en formación de todo el mundo pudieron disfrutar de diferentes ponencias en vivo o sobre demanda. Para aquellos que se registraron siguen disponibles en su sitio web múltiples ponencias de diferentes temas y ramas de la radiología ${ }^{22}$.

El American College of Radiology cuenta con un sitio web destinado de forma específica a recolectar material informativo acerca del SARS-COV-2 y la pandemia actual; su propósito es ayudar a la comunidad radiológica a proteger a sus pacientes y a sí misma. De manera adicional, alienta a sus miembros a enviar sus preguntas referentes al aspecto clínico de la infección por COVID-19. Más información se halla en https://www.acr. org / Clinical-Resources / COVID-19Radiology-Resources ${ }^{23}$

Aunque existen muchos beneficios potenciales de estas nuevas herramientas de aprendizaje a distancia, la enseñanza centrada en el alumno por parte de mentores experimentados es todavía una parte fundamental del proceso de aprendizaje. Es por ello fundamental que los educadores se familiaricen con los futuros recursos educativos en radiología para utilizarlos de forma innovadora y creativa; de ese modo no se perderá el aspecto interactivo de la educación médica y se avanzará al mismo paso que la tecnología. Hoy en día se halla bajo estudio la utilidad de la inteligencia artificial dentro de las actividades docentes ${ }^{13}$ y de formación de médicos radió$\log$ os $\mathrm{y}$, si bien el tema rebasa el alcance de este documento, es sin duda un tema a seguir de $\operatorname{cerca}^{24,25}$. 


\section{ENCUESTA EN LÍNEA DE EDUCACIÓN A DISTANCIA}

Con la única finalidad de conocer la forma en que se sucedieron los cambios y se implementaron con rapidez las soluciones tecnológicas en la educación para los radiólogos y los médicos en formación, se realizó una encuesta en el mes de junio del 2020, a tan sólo tres meses de establecerse el semáforo rojo en este país. Se obtuvieron respuestas de 182 personas, de las cuales una cuarta parte la representaron residentes del primer año $(26 \%)$, el $65 \%$ de la población se integró con residentes y el restante con médicos egresados y residentes de alta especialidad (Fig. 3). La plataforma más utilizada fue Zoom con 126 usuarios $(70 \%)$, seguida por Teams usada por 26 usuarios; otras plataformas fueron Webex, Skype, Google meet, Google classroom, y sólo dos usuarios mencionaron continuar con clases presenciales (Fig. 4).

El 70\% de los entrevistados consideró que el uso de plataformas en línea no sustituye a las clases educativas presenciales y que las áreas más afectadas fueron las que requieren uso de habilidades presenciales, como ultrasonido e intervención; se concluyó que la falta interacción es el peor aspecto del uso de las plataformas virtuales, según lo comentó el $60 \%$ de los entrevistados (Fig. 5), seguido por una disminución de la retroalimentación y la baja calidad de las presentaciones; sólo dos personas encuestadas no consideraron ningún aspecto negativo de la utilización de alternativas digitales. El porcentaje de aprendizaje apreciativo se redujo entre $25 \%$ y $75 \%$ entre los entrevistados, de los cuales sólo el 10\% refirió un porcentaje de

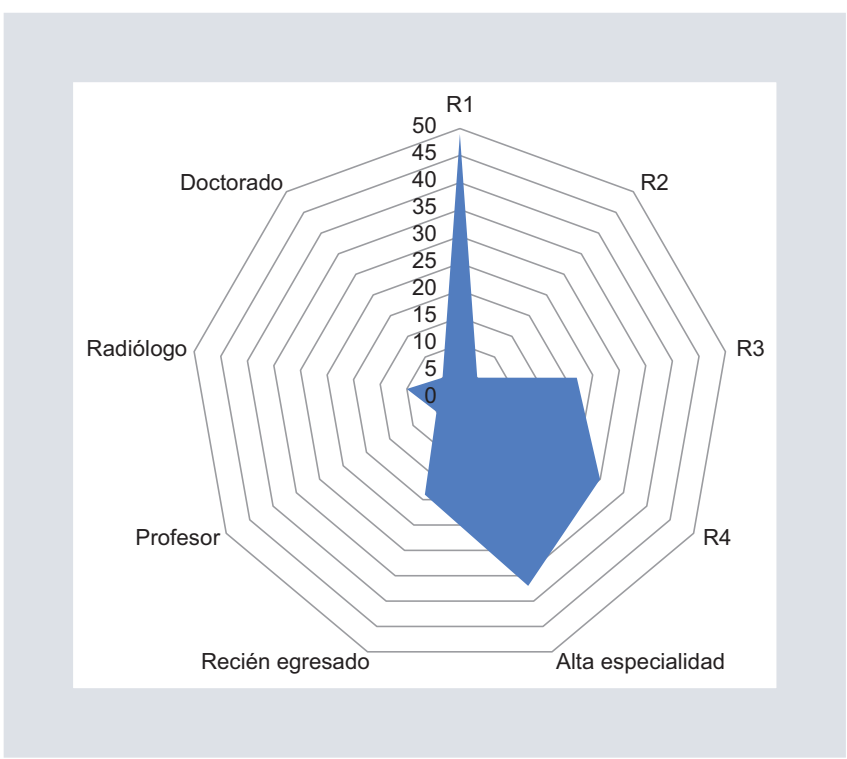

Figura 3. Distribución del grado académico de la población encuestada.

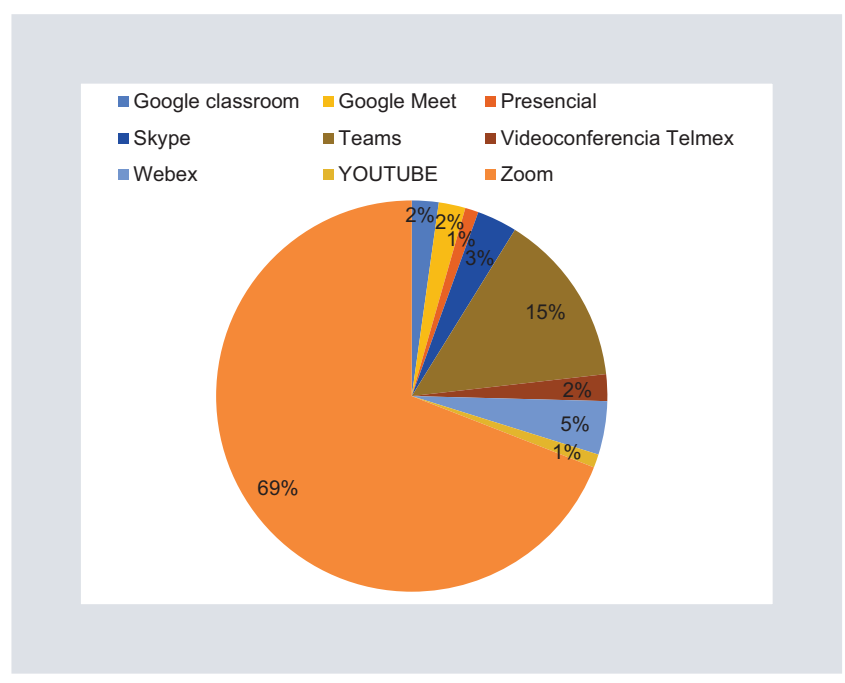

Figura 4. Porcentaje de plataformas más utilizadas para impartir clases virtuales durante la contingencia por COVID-19.

aprendizaje durante la contingencia cercano al $100 \%$.

\section{ANÁLISIS DE RESULTADOS}

Este estudio permitió describir las modificaciones necesarias de los protocolos de personal 


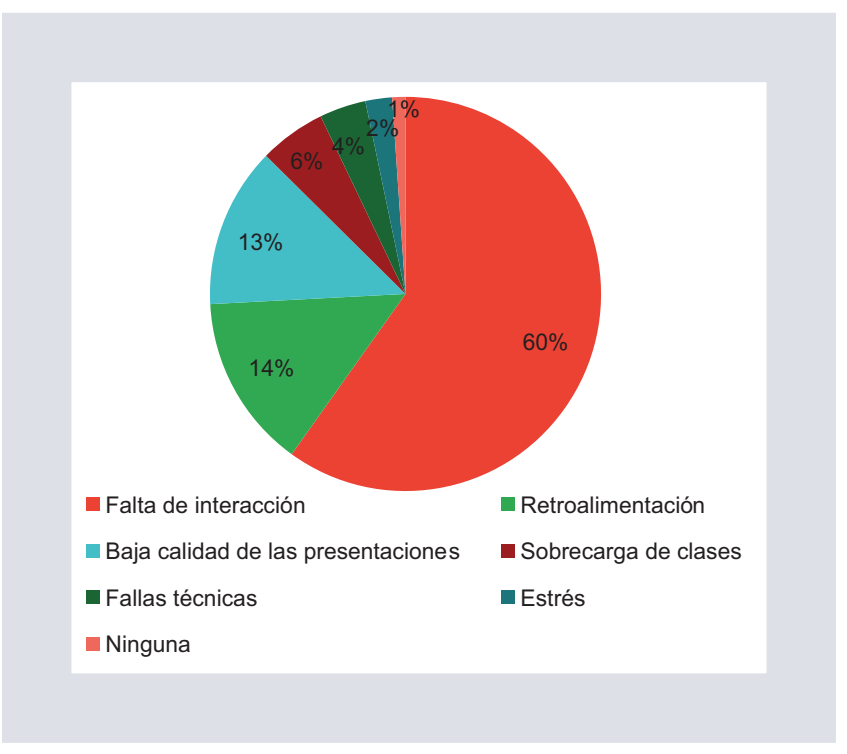

Figura 5. Aspectos negativos del uso de las plataformas en línea entre los entrevistados. Naranja: falta de interacción; azul: ausencia de retroalimentación; amarillo: baja calidad de las presentaciones.

médico, que continúa con actividades académicas de la especialidad de radiología e imagen, luego de los acontecimiento globales de la pandemia por COVID-19 y con el fin de disminuir las aglomeraciones. La mayor parte de las instituciones académicas optó por adoptar la utilización de plataformas digitales para continuar con la aplicación de clases, lo que impidió así una interrupción del aprendizaje. Sin embargo, esta medida representa nuevos retos y perspectivas de medidas $\mathrm{y}$ modificaciones inmediatas que no han valorado de manera certera el efecto de los elementos positivos y negativos en la educación actual.

Al realizar un interrogatorio dirigido de médicos residentes y adscritos del área de radiología, y tal y como sería previsible, las áreas más afectadas en aprendizaje son aquellas que requieren el aprendizaje técnico y práctico, como lo son los procesos de ultrasonido y la radiología intervencionista (Fig. 6). Esto se

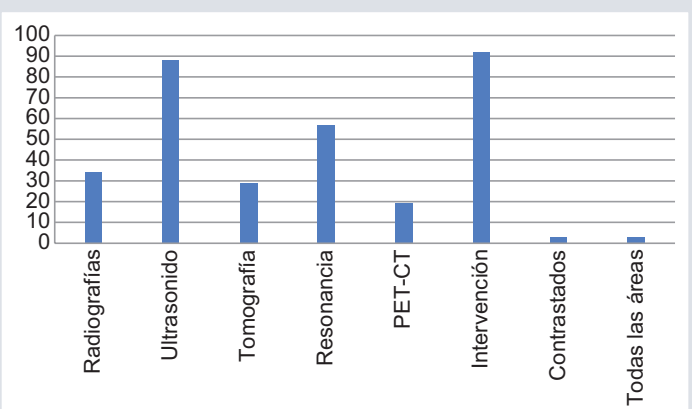

Figura 6. Áreas consideradas de mayor afectación académica en la contingencia por COVID-19.

debe a que, a pesar de conocer la teoría, en estos aspectos de la radiología son imprescindible la práctica y la supervisión dirigida, pero debe reconocerse que uno de los elementos más notables es la disminución del proceso de retroalimentación probablemente explicable por las dificultades en la utilización de plataformas, configuraciones de micrófonos, participaciones de discusión inmediatas con interacciones múltiples que se ven limitadas por la dificultar en encender y apagar los micrófonos, o artefactos acústicos producidos por múltiples personas que hablan al mismo tiempo. De cualquier forma, más de la mitad de los encuestados mostraron su preocupación por la disminución del proceso de retroalimentación en las clases virtuales respecto de las clases presenciales, según lo reflejaron de manera global los entrevistados, de los cuales la mayor parte considera que las plataformas virtuales no han logrado sustituir a las clases educativas presenciales; más aún, ha decrecido el aprendizaje de forma notoria entre 25\% y $75 \%$ según lo señala el $75 \%$ de los entrevistados.

En cuanto a las tecnologías empleadas, la mayor parte de los usuarios en este medio 
utilizó la plataforma de Zoom, algo promovido en su mayor parte por la gratuidad de la plataforma y la mayor promoción que tuvo académicamente como una herramienta para clases en línea durante la pandemia en diversas ramas educativas.

\section{CONCLUSIÓN}

Los tiempos en la radiología han cambiado y ante la emergencia sanitaria por COVID-19, la solución tecnológica más rápida ha sido la adopción de educación en línea, así como el uso de sistemas de gestión del aprendizaje en plataformas (LMS) y sistemas de transmisión de video en vivo como una forma de sustitución parcial de las clases presenciales para enfrentar la contingencia, sin afectar el aprendizaje. No obstante, se trata de una implementación forzada de estas estrategias, sin estudios previos que demuestren las áreas de ventaja y desventaja, lo cual no ha permitido generar un análisis de la aplicación de programas de aprendizaje virtual. Este trabajo ha demostrado que la forma de aplicación actual no sustituye de manera completa a las clases presenciales y ello ha generado áreas de afectación, sobre todo en zonas de destrezas de técnicas requeridas y además ha disminuido conceptos clave apreciativos, como la sensación de aprendizaje fomentado. Así se identificó debido a una menor retroalimentación y participación de los profesores que dirigen el aprendizaje, así como de los estudiantes asistentes e incluso aspectos preocupantes como la disminución de la calidad de las clases, con una consideración prácticamente nula de igualdad entre clases virtuales y presenciales. Identificar estos aspectos permite el conocimiento de nichos de oportunidad en los que pueden mejorarse otras alterativas que amortigüen el efecto negativo en la educación de los profesionales médicos. Sin duda, el vínculo personal no debe perderse dada la importancia de mantener la práctica y supervisión dirigida entre maestros y los residentes en formación, toda vez que gran parte del aprendizaje final siempre recaerá sobre la retroalimentación que éstos ofrezcan gracias a la experiencia. No debe olvidarse que las alternativas escogidas varían y que siempre dependen del grado de virtualidad de sus programas.

\section{AGRADECIMIENTOS}

Los autores agradecen a todos los radiólogos $y$ residentes que se han quedado en el camino durante la pandemia por la enfermedad COVID-19. También agradecen a todos los médicos que apoyaron con la información obtenida para esta investigación.

\section{CONFLICTO DE INTERESES}

Los autores declaran no tener conflicto de intereses.

\section{FINANCIAMIENTO}

El financiamiento del presente trabajo corrió por cuenta de los autores.

\section{RESPONSABILIDADES ÉTICAS}

Protección de personas y animales. Los autores declaran que para esta investigación no 
se han realizado experimentos en seres humanos ni en animales.

\section{Confidencialidad de los datos. Los autores declaran que en este artículo no aparecen da- tos de pacientes.}

\section{Derecho a la privacidad y consentimiento informado. Los autores declaran que en este artículo no aparecen datos de pacientes.}

\section{BIBLIOGRAFIÍA}

1. Sociedad Española de Radiología Médica. La radiología desde la aparición de la infección COVID-19. Análisis y recomendaciones. Abril, 2020. 1era edición, pp. 1-112. Disponible en: https://www.seram.es/images/site/ Futuro_Radiologia_COVID_SERAM_2.pdf

2. Emanuel EJ. The inevitable reimagining of medical education. JAMA. 2020;323(12):1127-1128. doi:10.1001/jama.2020.1227

3. Cornelio-Rodríguez G, Vadillo-Santos A, Pérez-Ladrón de Guevara P. Protocolo interhospitalario de actuación en las salas de radiología intervencionista durante la COVID-19. Anales de Radiología de México. 2020;19:1-14. Disponible en DOI:10.24875/ARM.20000073

4. Alvin MD, George E, Deng F. The impact of COVID-19 on radiology trainees. Radiology. 2020;296(2):246-248. Disponible: https://doi.org/10.1148/ radiol.2020201222

5. Mian A, Khan S. Medical education during pandemics: a UK perspective. BMC Medicine. 2020;18:100. Disponible en: https://doi.org/10.1186/ s12916-020-01577

6. Organización de las Naciones Unidas para la Educación, la Ciencia y la Cultura. COVID-19 y educación superior: de los efectos inmediatos al día después. Mayo 2020, pp. 1-57. Disponible: http:/ /www.iesalc.unesco.org/ wp-content/uploads/2020/05/COVID-19-ES-130520.pdf

7. Ros-Mendoza LH, Navarro-Monforte Y, Rambla-Sanz T. La enseñanza en radiología: un nuevo método para planificar y evaluar por competencias. Revista Argentina de Radiología. 2017;81(4):279-284. https://doi. org/10.1016/j.rard.2017.02.002
8. Paricio-Royo J. Un modelo de guía docente desde los resultados de aprendizaje y su evaluación. Zaragoza: Universidad de Zaragoza, Instituto de Ciencias de la Educación; 2011

9. Consejo Mexicano de Radiología e Imagen A.C. Consultado el 2 de enero 2021. Disponible en: https://www.cmri.org.mx/bienvenidacmri2.php

10. Medical Simulator. Habilidades técnicas, radiografía. Consultado el 10 de enero 2021. Disponible en: https://medical-simulator.com/Radiografia-131804

11. Medical Simulator. Habilidades técnicas, radiografía. Consultado el 10 de enero 2021. Disponible en: https://medical-simulator.com/radiologiaintervencionista/4377-1-CAE-CathLabVR.html

12. Scarsbrook A, Graham R, Perriss R. Radiology education: a glimpse into the future. Clinical Radiology. 2006;640-648.

13. Shi J, Miskin N, Dabiri B, DeSimone A, Schaefer P, Bay C, et al. Quantifying impact of disruption to radiology education during the COVID-19 pandemic and implications for future training. Diagnostic Radiology. 2020;1-5.

14. Sparacia G, Cannizzaro F, D'Alessandro D, D'Alessandro M, Caruso G, Lagalla R. Informatic in radiology. Radiographics. 2007;573-581.

15. Simpson S, Cook T. Artificial intelligence and the trainee experience in radiology. J Am Coll Radiol. 2020;1388-1393.

16. Radiopaedia. 01 de enero de 2021. Obtenido de Radiopaedia: https:// radiopaedia.org

17. CTisus. (01 de enero de 2021). Obtenido de CTisus everything you need to know about computed tomography (CT) \& CT scanning: https://www. ctisus.com/responsive/default.asp

18. Elsevier. (01 de enero de 2021). STATdx. Obtenido de STATdx: https:/ /app. statdx.com

19. International Society of Radiology. (2021). Obtenido de ICRE International Commission on Raidiology Education: https://www.isradiology.org/ education-icre

20. Colegio Interamericano de Radiología. Obtenido del Colegio Interamericano de Radiología CIR: https://www.webcir.org/index.php.

21. ESR Connect European Society of Radiology. Obtenido de ECR Connect: https:// connect.myesr.org

22. Radiological Society of North America RSNA. Obtenido de RSNA Radiological Society of North America: https://www.rsna.org/membership

23. American College of Radiology ACR. Obtenido ACR COVID-19 Clinical Resources for Radiologists: https://www.acr.org/Clinical-Resources/ COVID-19-Radiology-Resources.

24. Elsevier. Beyond business as usual: radiology residency educational response to the COVID-2019 pandemic. Clinical Imaging. 2021;349-353.

25. Kelly A, Mullan P. Teaching and assessing professionalism in radiology: resources and scholarly opportunities to contribute to required expectations. Academic Radiology. 2018;599-609. 